\title{
Advantages of Digital Subtraction Angiography During Nerve Block
}

\author{
Sun kyung Park ${ }^{1}$; Yun Suk Choi ${ }^{1, *}$ \\ ${ }^{1}$ Department of Anesthesiology and Pain Medicine, Jeju National University Hospital, School of Medicine, Jeju National University, Jeju, Korea \\ ${ }^{*}$ Corresponding author: Yun Suk Choi, Department of Anesthesiology and Pain Medicine, Jeju National University Hospital, School of Medicine, Jeju National University, P. O. Box: \\ 690-716, Jeju, Korea. Tel: +82-647172026, Fax: +82-647172042,, E-mail: solafide5@yahoo.co.kr
}

Received: May 2, 2014; Revised: June 1, 2014; Accepted: June 12, 2014

\begin{abstract}
Introduction: Most pain physicians know that fluoroscopy might improve the safety, accuracy, and efficacy of nerve block; however, it is difficult to distinguish the previously administered contrast medium from the injecting contrast medium, and to identify accurate contrast medium diffusion flow in a case of existing radiodensities such as cement and screw. Digital subtraction angiography (DSA) can improve accuracy of nerve blocks.

Case Presentation:We described our experiences with two successful transforaminal epidural injections and nerve block of two patients with 73 and 68 years of age who were diagnosed as failed back surgery syndrome.

Conclusions: The advantages of DSA in fluoroscopy-guided transforaminal epidural block and nerve block are identification of the degree of appropriate contrast flow (epidural and nerve root sleeve) and the intravascular administration without overlapping radiodense structures.
\end{abstract}

Keywords:Digital Subtraction Angiography; Nerve Block; Transforaminal Epidural Block

\section{Introduction}

Nerve block is the most fundamental used method in pain clinics. Although nerve block has been performed with blind technique, fluoroscopy- or ultrasound-guided nerve blocks are mainly used due to inaccuracy and complications of nerve block with blind technique. The diffusion of the injected drug can be forecasted by administering a contrast medium in fluoroscopy-guided nerve block; however, when a contrast medium is injected repeatedly, distinguishing the previously administered contrast medium flow from the existing contrast medium is difficult by fluoroscopy alone. Several studies have reported that the use of digital subtraction angiography (DSA) increases the detection rate of the intravascular injection frequencies (1-3). However, a recent study has suggested limitations to DSA because of associated fatal complications despite the absence of intravascular injection in digital subtraction fluoroscopy (4). There was no study about advantage of DSA in a case of radiodensity during nerve block. Therefore, we aimed to examine the benefits of DSA by introducing our experience with accurate contrast medium diffusion through DSA in a case of radiodensity including bone cement and screw.

\section{Case Presentation}

The C-arm used in this case report was installed using DSA software (GE OEC 9900 elite, GE Healthcare, Salt Lake City, Utah, USA).

\subsection{Case 1}

A 73-year-old female underwent L4-L5 fixation under a diagnosis of lumbar spinal stenosis in 2004, which was followed by vertebroplasty at L1 and L2 due to vertebral compression fracture. She visited our hospital due to severe low back pain (numerical rating scale [NRS], 9-8/10) that radiated down to the right lower extremity. She was diagnosed with failed back surgery syndrome (FBSS) and took medications and used fentanyl patch $(25 \mu \mathrm{g} / \mathrm{h})$. Caudal epidural block, lumbar medial branch block, and thoracic epidural block were performed. To control the pain radiating to the right lower extremity, a transforaminal epidural steroid injection (TFESI) at the right-sided L5 nerve was arranged; the C-arm-guided image is shown in Figure $1 \mathrm{~A}$. Conventional C-arm fluoroscopy did not distinguish between epidurogram and intravascular uptake after injecting contrast medium because of the instrumentation. An anteroposterior digital subtraction image shows a simultaneous epidural and vascular contrast pattern (Figure $1 \mathrm{~B}$ ). Therefore, after redirecting block needle and injecting contrast, we checked the epidurogram without intravascular pattern. The spread of contrast medium and intravascular contrast medium were confirmed by DSA, and an L5 TFESI (dexamethasone, $5 \mathrm{mg}$ ) was performed on the right side. The treatment decreased right lower extremity pain significantly (NRS, 2/10).

Copyright (C) 2014, Iranian Society of Regional Anesthesia and Pain Medicine (ISRAPM); Published by Kowsar. This is an open-access article distributed under the terms of the Creative Commons Attribution-NonCommercial 4.0 International License (http://creativecommons.org/licenses/by-nc/4.0/) which permits copy and redistribute the material just in noncommercial usages, provided the original work is properly cited. 
Park Sk et al.

Figure 1. Right L5 Transforaminal Epidural Steroid Injection Plain Anteroposterior View
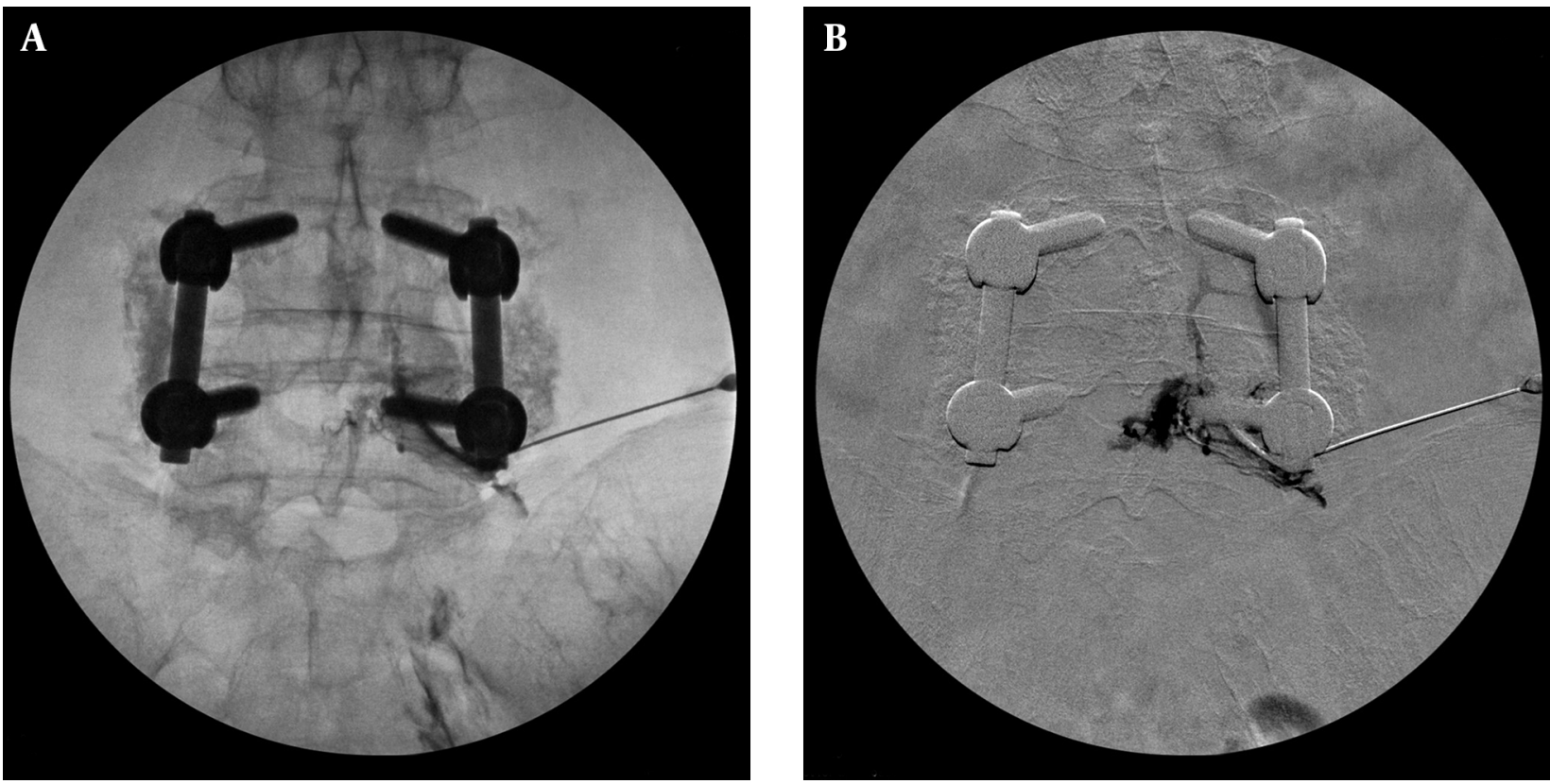

A, Live fluoroscopic image taken during contrast injection. B, Digital subtraction image taken during same injection

Figure 2. Right L5 Transforaminal Epidural Steroid Injection Plain Anteroposterior View
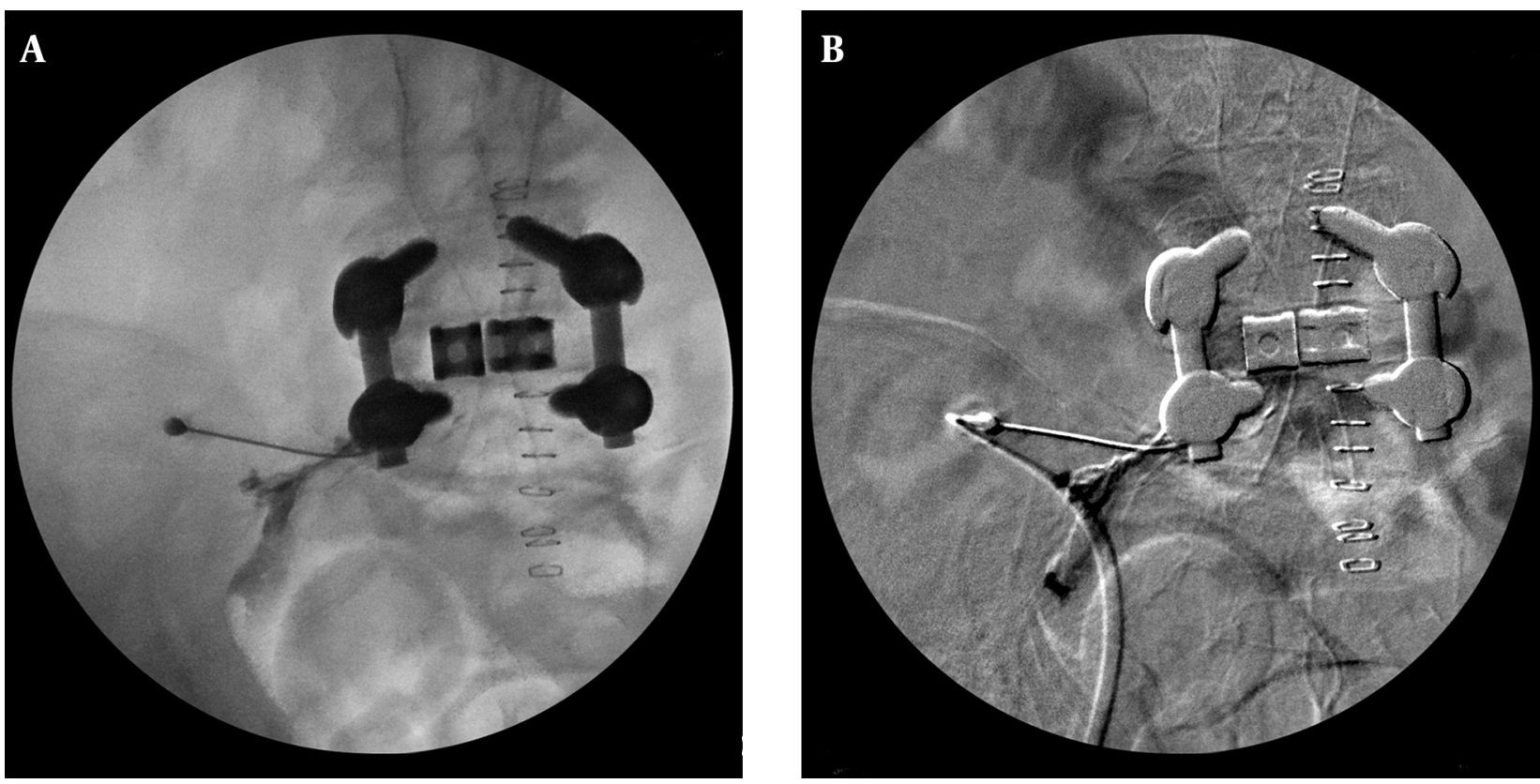

A, Live fluoroscopic image taken during contrast injection. B, Digital subtraction image taken during the same injection.

\subsection{Case 2}

A 68-year-old female underwent L5-S1 fixation and then nerve block was requested from the department of neurosurgery due to postoperative pain in low back, left sacral region, and lower extremities. The left-sided L5 TFESI was planned under a diagnosis of FBSS. The C-arm was rotated obliquely until the "Scotty dog" view was obtained. Using an oblique approach, a 100-mm 22-G needle was advanced toward the intervertebral foramen. The needle depth was 
Park Sk et al.

monitored with a lateral view. The needle placement was at one-half to one-third of the interval between the vertical edges of the vertebral body. As shown in Figure $2 \mathrm{~A}$, due to instrumentation, distinguishing contrast diffusion from conventional C-arm fluoroscopy was difficult. Following the injection of contrast medium, a digital subtraction image demonstrated correct needle position and dye flow alongside the exiting nerve root and epidural space. As shown in Figure 2 B, left L5 TFESI (dexamethasone, $5 \mathrm{mg}$ ) was performed after examining the pattern of the contrast diffusion and the absence of intravascular injection by DSA. Consequently, the sacral and left lower extremity radiating pain decreased by more than $50 \%$.

\section{Discussion}

Most interventional pain physician are comfortable with using fluoroscopy and understand its importance in improving the safety, accuracy, and efficacy of diagnostic and therapeutic procedures. The addition of digital subtraction may enhance the use of radiography and the accuracy of our interpretation. DSA is a powerful fluoroscopy technique to clearly visualize blood vessels in interventional radiology. It requires the subtraction of high-contrast structures to observe radiographic contrast media in blood vessels. Subtraction methods, which are used to remove distracting structures such as soft tissue and bone in DSA, are time (temporal subtraction) and energy related (energy subtraction). In addition, hybrid subtraction is performed by utilizing the merits of temporal and energy subtractions (5). In this study, contrast diffusion and intravascular administration were clearly determined in cement and instrument subtracted images when the contrast flow spread was unclear; cement and instruments showed higher radiodensity than soft tissue and bone structures did. In the process of C-armguided nerve block, the incidence of accidental intravascular penetration was reported to be $30.7 \%$, with incidences of $9.9 \%$ at the lumbar level and $63.4 \%$ at the cervical level (6). By enhancing the ability of recognizing intravascular injection by digital subtraction fluoroscopy and live injection observation, the frequency of intravascular complications might be reduced (1). According to a previous study on 134 patients, the prevalence of intravascular injection during CTFESI was $17.9 \%$ with fluoroscopy alone and $32.8 \%$ with DSA (7). DSA can visualize more details of intravascular injections.

The numbers of patients with FBSS is increasing along with the number of spinal surgeries (8). During nerve blocks in FBSS (7), the administered drugs do not spread well because of epidural vessel engorgement at the surgery site and adjacent areas (9). Moreover, intravascular injection is often hardly distinguishable after dye injection due to radiodense instrumentation. In the case of vertebroplasty, intravascular penetration is often hardly distinguishable during dye injection due to radiodense bone cement. Therefore, more detailed radiographic im- ages should be obtained with DSA during nerve block, particularly in patients with FBSS or those who had undergone vertebroplasty, when contrast medium images are difficult to interpret due to artifacts including instrumentation or bone cement and fluoroscopic image is distracted due to repeated administration of contrast medium during epidurography. In these case reports, intravascular injections were difficult to be detected with a fluoroscopic dye pattern due to radiodense instrumentation. However, we successfully performed nerve blocks by determining the extent of intravascular penetration in the epidural space by applying digital subtraction fluoroscopy and thoroughly observing the dye spread. Therefore, DSA improves accuracy during nerve blocks when the patients have the radiodense structures such as bone cement and spine instrument. It can be an advantage of DSA during nerve blocks.

We have presented our experience of DSA. There have been a few studies showing DSA's ability to distinguish the vascular uptake and contrast flow while performing nerve blocks when contrast medium was injected $(1,5,10)$. However, none of those studies focused on performing the block with DSA on patients with radiodense structures. Therefore, further randomized controlled studies are needed to demonstrate the benefits of DSA. In addition, the shortcomings of DSA are an increase in radiation exposure, reduced image quality due to motion artifacts, the long duration of the procedure because of repeated performances, and the use of higher doses of contrast agent (8). In addition, the installation of DSA facility entails considerable expenses (1). Although DSA is less cost-effective than conventional fluoroscopy is, this case report shows clear benefits of performing the nerve blocks with DSA in patients with radiodense structures. The advantages are increased accuracy of the needle position and better distinguishable contrast flow.

In conclusion, the advantages of DSA in fluoroscopyguided transforaminal epidural block and nerve block are identification of the degree of appropriate contrast flow (epidural and nerve root sleeve) and the intravascular administration without overlapping radiodense structures.

\section{Acknowledgements}

We would like to thank the department of Anesthesiology and Pain Medicine of Jeju National University Hospital.

\section{Authors' Contributions}

All authors contributed to patient care and preparation of this manuscript.

\section{References}

1. Jasper JF. Role of digital subtraction fluoroscopic imaging in detecting intravascular injections. Pain Physician. 2003;6(3):369-72.

2. Chang Chien GC, Candido KD, Knezevic NN. Digital subtraction 
angiography does not reliably prevent paraplegia associated with lumbar transforaminal epidural steroid injection. Pain Physician. 2012;15(6):515-23.

3. Kim do W, Han KR, Kim C, Chae YJ. Intravascular flow patterns in transforaminal epidural injections: a comparative study of the cervical and lumbar vertebral segments. Anesth Analg. 2009;109(1):233-9.

4. Furman MB, Giovanniello MT, O'Brien EM. Incidence of intravascular penetration in transforaminal cervical epidural steroid injections. Spine (Phila Pa 1976). 2003;28(1):21-5.

5. McLean JP, Sigler JD, Plastaras CT, Garvan CW, Rittenberg JD. The rate of detection of intravascular injection in cervical transforaminal epidural steroid injections with and without digital subtraction angiography. PM R. 2009;1(7):636-42.

6. Ionasec RI, Heigl B, Hornegger J. Acquisition-related motion compensation for digital subtraction angiography. Comput Med Imaging Graph. 2009;33(4):256-66.

7. Takeshima N, Miyakawa H, Okuda K, Hattori S, Hagiwara S, Takatani J, et al. Evaluation of the therapeutic results of epiduroscopic adhesiolysis for failed back surgery syndrome. BrJAnaesth. 2009;102(3):400-7.

8. Hussain A, Erdek M. Interventional pain management for failed back surgery syndrome. Pain Pract. 2014;14(1):64-78.

9. Helm Ii S, Benyamin RM, Chopra P, Deer TR, Justiz R. Percutaneous adhesiolysis in the management of chronic low back pain in post lumbar surgery syndrome and spinal stenosis: a systematic review. Pain Physician. 2012;15(4):E435-62.

10. Lee MH, Yang KS, Kim YH, Jung HD, Lim SJ, Moon DE. Accuracy of live fluoroscopy to detect intravascular injection during lumbar transforaminal epidural injections. Korean J Pain. 2010;23(1):18-23. 